Journal for ImmunoTherapy of Cancer

\title{
Axitinib plus avelumab in the treatment of recurrent glioblastoma: a stratified, open-label, single-center phase 2 clinical trial (GliAvAx)
}

Gil Awada (1) , ${ }^{1}$ Laila Ben Salama, ${ }^{1}$ Jennifer De Cremer, ${ }^{2}$ Julia Katharina Schwarze, ${ }^{1}$ Lydia Fischbuch, ${ }^{1}$ Laura Seynaeve, ${ }^{3}$ Stephanie Du Four, ${ }^{4}$ Anne-Marie Vanbinst, ${ }^{5}$ Alex Michotte, ${ }^{6}$ Hendrik Everaert, ${ }^{7}$ Anne Rogiers, ${ }^{8}$ Peter Theuns, ${ }^{2}$ Johnny Duerinck, ${ }^{4}$ Bart Neyns ${ }^{1}$

\section{ABSTRACT}

To cite: Awada G, Ben Salama L, De Cremer J, et al. Axitinib plus avelumab in the treatment of recurrent glioblastoma: a stratified, openlabel, single-center phase 2 clinical trial (GliAvAx). Journal for ImmunoTherapy of Cancer 2020;8:e01146. doi:10.1136/ jitc-2020-001146

GA and LBS contributed equally.

Accepted 16 September 2020

Check for updates

(c) Author(s) (or their employer(s)) 2020. Re-use permitted under CC BY-NC. No commercial re-use. See rights and permissions. Published by BMJ.

For numbered affiliations see end of article.

Correspondence to

Prof. Dr. Bart Neyns;

bart.neyns@uzbrussel.be against rGB. events.
Background No treatment demonstrated to improve survival in patients with recurrent glioblastoma ( $\mathrm{rGB}$ ) in a randomized trial. Combining axitinib with the programmed cell death ligand 1 blocking monoclonal antibody avelumab may result in synergistic activity

Methods Adult patients with rGB following prior surgery, radiation therapy and temozolomide chemotherapy were stratified according to their baseline use of corticosteroids. Patients with a daily dose of $\leq 8 \mathrm{mg}$ of methylprednisolone (or equivalent) initiated treatment with axitinib (5 mg oral two times per day) plus avelumab (10 mg/kg intravenous every 2 weeks) (Cohort-1). Patients with a higher baseline corticosteroid dose initiated axitinib monotherapy; avelumab was added after 6 weeks of therapy if the corticosteroid dose could be tapered to $\leq 8 \mathrm{mg}$ of methylprednisolone (Cohort-2). Progression-free survival at 6 months (6-m-PFS\%), per immunotherapy response assessment for neuro-oncology criteria, served as the primary endpoint.

Results Between June 2017 and August 2018, 54 patients (27 per cohort) were enrolled and initiated study treatment (median age: 55 years; $63 \%$ male; $91 \%$ Eastern Cooperative Oncology Group Performance Status 0-1). Seventeen $(63 \%)$ patients treated in Cohort-2 received at least one dose of avelumab. The $6-\mathrm{m}-\mathrm{PFS} \%$ was $22.2 \%$ (95\% Cl 6.5\% to $37.9 \%)$ and $18.5 \%$ (95\% Cl $3.8 \%$ to $33.2 \%)$ in Cohort-1 and Cohort-2, respectively; median overall survival was 26.6 weeks ( $95 \% \mathrm{Cl} 20.8$ to 32.4 ) in Cohort- 1 and 18.0 weeks $(95 \% \mathrm{Cl} 12.5$ to 23.5$)$ in Cohort-2. The best objective response rate was $33.3 \%$ and $22.2 \%$ in Cohort-1 and Cohort-2, respectively, with a median duration of response of 17.9 and 19.0 weeks. The most frequent treatment-related adverse events were dysphonia $(67 \%)$, Iymphopenia $(50 \%)$, arterial hypertension and diarrhea (both 48\%). There were no grade 5 adverse

Conclusion The combination of avelumab plus axitinib has an acceptable toxicity profile but did not meet the prespecified threshold for activity justifying further investigation of this treatment in an unselected population of patients with rGB.

\section{INTRODUCTION}

Despite upfront multimodality treatment, progression will occur in more than half of all patients with glioblastoma (GB) (WHO grade IV glioma, GB) within less than 9 months from treatment initiation and less than $10 \%$ of patients will be alive 5 years after the initial diagnosis. ${ }^{1}$ At tumor progression following first-line treatment, no salvage therapeutic option demonstrated to significantly improve overall survival (OS) in a randomized clinical trial. Cytotoxic salvage therapies resulted in a best objective response rate (ORR) of 5\%-10\%, 6-month-progression-free survival rates (6-m-PFS\%) of $9 \%-21 \%$ and a median OS of 25-30 weeks. ${ }^{23}$

GB is characterized by profound neoangiogenesis, a cancer-associated physiopathological process in which the vascular endothelial growth factor (VEGF) signaling pathway plays a major role. VEGF is upregulated in GB by tissue hypoxia via the hypoxia-inducible factor 1-alpha pathway. By binding to its tyrosine kinase cell surface receptors (VEGFR-1, VEGFR-2 and VEGFR-3), VEGF has a mitogenic effect on endothelial cells and increases endothelial permeability leading to GB-associated edema. Moreover, autocrine VEGFR-1 and VEGFR-2 signaling promotes cancer cell survival in human GB models. ${ }^{4}$ Bevacizumab, a VEGF-neutralizing monoclonal antibody, demonstrated activity as a single agent with ORRs in the range of $28 \%-35 \%$ in recurrent GB (rGB), median PFS of 11-17 weeks and median OS of 26-37 weeks. ${ }^{5}$ Axitinib is an orally available, high-affinity tyrosine kinase inhibitor of the VEGF-receptors that is approved as a monotherapy for the treatment of patients with metastatic renal cell carcinoma who failed one prior line of systemic 
therapy. ${ }^{6}$ Axitinib has an antiangiogenic and survival prolongation effect in preclinical orthotopic GB models and inhibits tumor growth in a GB xenograft model with primary resistance to bevacizumab. ${ }^{7}$ Our group previously reported that axitinib monotherapy demonstrated antitumor activity (ORR 28\%) with manageable toxicity in $\mathrm{rGB}^{8}{ }^{8}$ In this non-comparative randomized phase 2 clinical trial, survival (6-m-PFS\% of $34 \%$ (95\% CI $14 \%$ to $54 \%$ )) was comparable to the survival of patients treated with bevacizumab. In a subsequent randomized phase 2 clinical trial, adding lomustine to axitinib did not improve survival as compared with treatment initiation with axitinib alone. ${ }^{9}$

Inhibition of the programmed cell death protein 1 (PD-1, CD279) or its ligand (PD-L1, CD274, B7 homolog 1 (B7-H1)) demonstrated clinical benefit across a large number of tumor types. ${ }^{10}$ In patients with rGB however, nivolumab, an $\operatorname{IgG}_{4}$ PD-1 blocking monoclonal antibody, failed to improve survival of patients with $\mathrm{rGB}$ when compared with bevacizumab (CheckMate-143 trial). ${ }^{11}$ Nivolumab treatment resulted in an ORR of $7.8 \%(95 \%$ CI $4 \%$ to $13 \%$ ) and a $6-\mathrm{m}-\mathrm{PFS} \%$ of $15.7 \%$ (95\% CI $10 \%$ to $21 \%$ ). The duration of response (DOR) on nivolumab was longer as compared with bevacizumab (11.1 vs 5.3 months).

VEGF contributes to suppression of antitumor immunity within the tumor microenvironment, while axitinib increases the infiltration of immune cells and reduces the suppressive capacity of monocytic myeloid-derived suppressor cells in an intracranial mouse melanoma model. ${ }^{12}$ Moreover, disease progression in rGB patients treated with axitinib was associated with increased regulatory T-cell numbers and T-cell exhaustion. ${ }^{13}$ Combination of VEGFR-inhibition by axitinib and PD-L1 blockade by avelumab (a PD-L1 blocking IgG I $_{1}$ monoclonal antibody) was found tolerable and significantly improved PFS in patients with untreated metastatic renal cell carcinoma. ${ }^{14}$ We, therefore, investigated and report in this manuscript the antitumor activity of the combination of axitinib plus avelumab for the treatment of patients with rGB. Given the negative impact of corticosteroid therapy, often used to control for GB-associated neurological symptoms, the question was addressed separately in two strata according to the baseline (BL) use of corticosteroids at therapeutic dose levels. ${ }^{15}$

\section{PATIENTS AND METHODS \\ Study design and participants}

This single-center, stratified, dual-stratum, open-label phase 2 clinical trial was conducted at the Universitair Ziekenhuis Brussel (Brussels, Belgium) in patients aged 18 years or older with a recurrence of previously histologically confirmed GB/gliosarcoma (regardless of WHO grade at first diagnosis of glioma) following prior treatment with at least surgery (biopsy and/or resection), radiation therapy and temozolomide chemotherapy. Prior therapies for rGB were permitted (without a defined maximal number of prior treatment lines). The recurrence had to be documented as a measurable lesion on gadoliniumenhanced T1 MRI and as an enhancing lesion compared with normal brain tissue on 18-fluoroethyl-L-tyrosine positron emission tomography/CT $\left({ }^{18}\right.$ F-FET-PET/CT $)$. Eligible patients must have had an Eastern Cooperative Oncology Group Performance Status (ECOG PS) of 0-2, an estimated life expectancy of at least 3 months and an adequate organ function. Patients were excluded if they had received prior immunotherapy with an anti-PD-1, -PDL1/2, -CD137 or -CTLA4 (cytotoxic T-lymphocyteassociated antigen 4) antibody or any other drug specifically targeting T-cell costimulation or if they had received prior therapy with axitinib or other drugs targeting the VEGF-pathway (such as bevacizumab). Other inclusion/ exclusion criteria can be found in the online supplementary table S1.

All participants provided written informed consent.

\section{Procedures}

Patients were screened for eligibility by history, physical examination, blood and urinary analysis (including blood chemistry, hematological, endocrinological tests and dipstick proteinuria), electrocardiography, MRI of the brain (including T1 1 gadolinium contrast, T2/ FLAIR, diffusion/perfusion and spectroscopy), and ${ }^{18}$ F-FET-PET/CT of the brain.

Patients were stratified according to their BL use of corticosteroids. Patients without need of or on a physiological dose of corticosteroids (8 mg methylprednisolone (equivalent of $1.5 \mathrm{mg}$ dexamethasone) or less) initiated axitinib at an oral dose of $5 \mathrm{mg}$ two times a day, and avelumab $(10 \mathrm{mg} / \mathrm{kg}$ administered intravenously over $60 \mathrm{~min}$ every 2 weeks) (Cohort-1). Administration of avelumab was preceded by administration of $1 \mathrm{~g}$ of paracetamol and 5 $\mathrm{mg}$ of levocetirizine, as indicated by the label.

Patients who were treated at BL with a supraphysiological dose of corticosteroids initiated axitinib $5 \mathrm{mg}$ two times a day. Avelumab $10 \mathrm{mg} / \mathrm{kg}$ was added to axitinib after 6 weeks if the dose of corticosteroids could be tapered to a daily equivalent dose of methylprednisolone $8 \mathrm{mg}$ or less (Cohort-2). The dose of axitinib could be increased to $10 \mathrm{mg}$ two times a day or decreased to $1 \mathrm{mg}$ two times a day according to tolerance.

Throughout the course of the study, patients were evaluated on a continuous basis every 2 weeks with a clinic examination and blood analysis. Every 6 weeks, tumor response assessments were performed based on gadolinium-enhanced MRI of the brain. Follow-up imaging with ${ }^{18}$ F-FET-PET/CT was scheduled as clinically indicated to complement MRI results.

In both cohorts, study therapy was continued until confirmed progression of disease, unacceptable toxicity or withdrawal of consent to continue study treatment. Patients were allowed to continue study treatment following the first documentation of disease progression if the investigator considered this to be in the best interest 
of the patient. Patients with confirmed tumor progression needed to stop study treatment.

\section{Endpoints}

The primary endpoint of the trial was the percentage of patients who were alive and free from progression at 6 months (24 weeks) following the date of treatment initiation (6-month-PFS rate, 6-m-PFS\%, estimated by the Kaplan-Meier method). Tumor response and progression of disease were defined according to the immunotherapy response assessment for neuro-oncology (iRANO) criteria. The antitumor effect of the combination therapy of avelumab with axitinib was estimated on both strata separately. Additional objectives and secondary endpoints were to estimate the median PFS/OS by Kaplan-Meier estimates and the tumor response according to iRANO criteria (assessed by the investigators); to document treatment disposition for avelumab and axitinib; to document the safety of avelumab and axitinib (defined and graded according to the Common Terminology Criteria for Adverse Events V.4.03) and to assess the neurocognitive function and health-related quality of life (HRQoL) (online supplementary table S2).

\section{Statistical analysis}

The sample size for Cohort-1 is determined according to a one-stage Fleming design. Avelumab plus axitinib was considered worthy of further investigations if a 6-m-PFS\% of $>50 \%$ is observed $(p(0)=0.30$ and $p(1)=0.50)$. With an alpha error of 0.10 , and a beta error of 0.20 , a sample size of 26 patients is required.

The outcome of patients recruited to Cohort-2 is considered to be of an exploratory nature and no predefined statistical hypothesis was used to calculate the sample size for this cohort separately. Recruitment of patients to Cohort-2 will be limited to the duration of recruitment of patients to the parallel Cohort- 1 or for a maximum number of 26 patients. This trial was, therefore, planned to recruit a maximum total of $2 \times 26$ patients (52 patients).

Statistical analyzes were performed using SPSS StatisticsV. 26.0 (SPSS). This study is registered with ClinicalTrials.gov, number NCT03291314.

\section{RESULTS}

\section{Patient BL characteristics}

Between June 142017 and August 29 2018, 55 patients were screened for eligibility. Fifty-four eligible patients were enrolled in the trial, 27 in each cohort (figure 1). BL characteristics are shown in table 1 . Sixty-three per cent of patients were male. The median age at treatment initiation was 57 years (range 20-70) in Cohort-1, and 47 years (range 19-75) in Cohort-2. Most patients had an

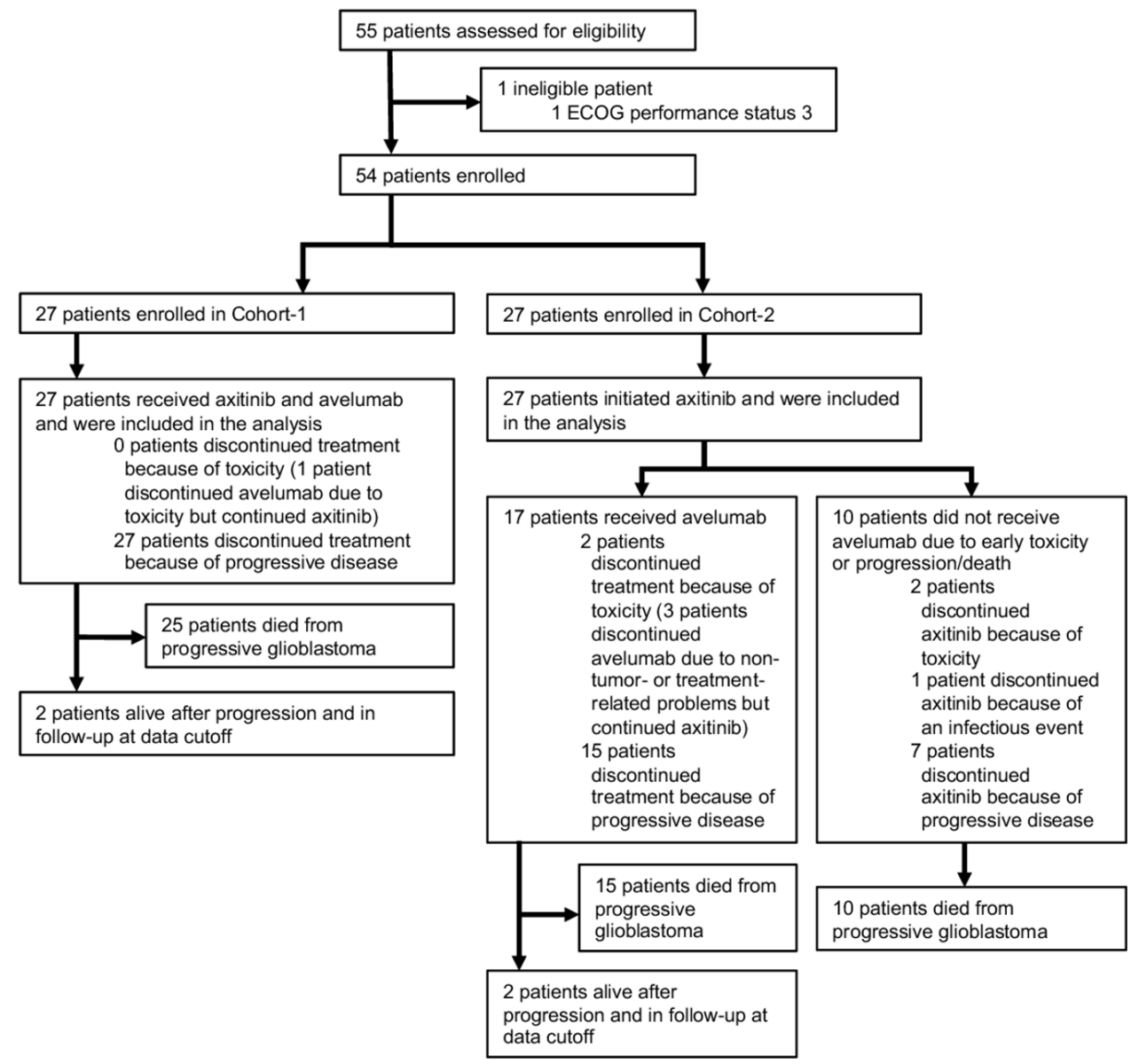

Figure 1 CONSORT-diagram. CONSORT, Consolidated Standards of Reporting Trials; ECOG, Eastern Cooperative Oncology Group. 
Table 1 Baseline characteristics of the study population

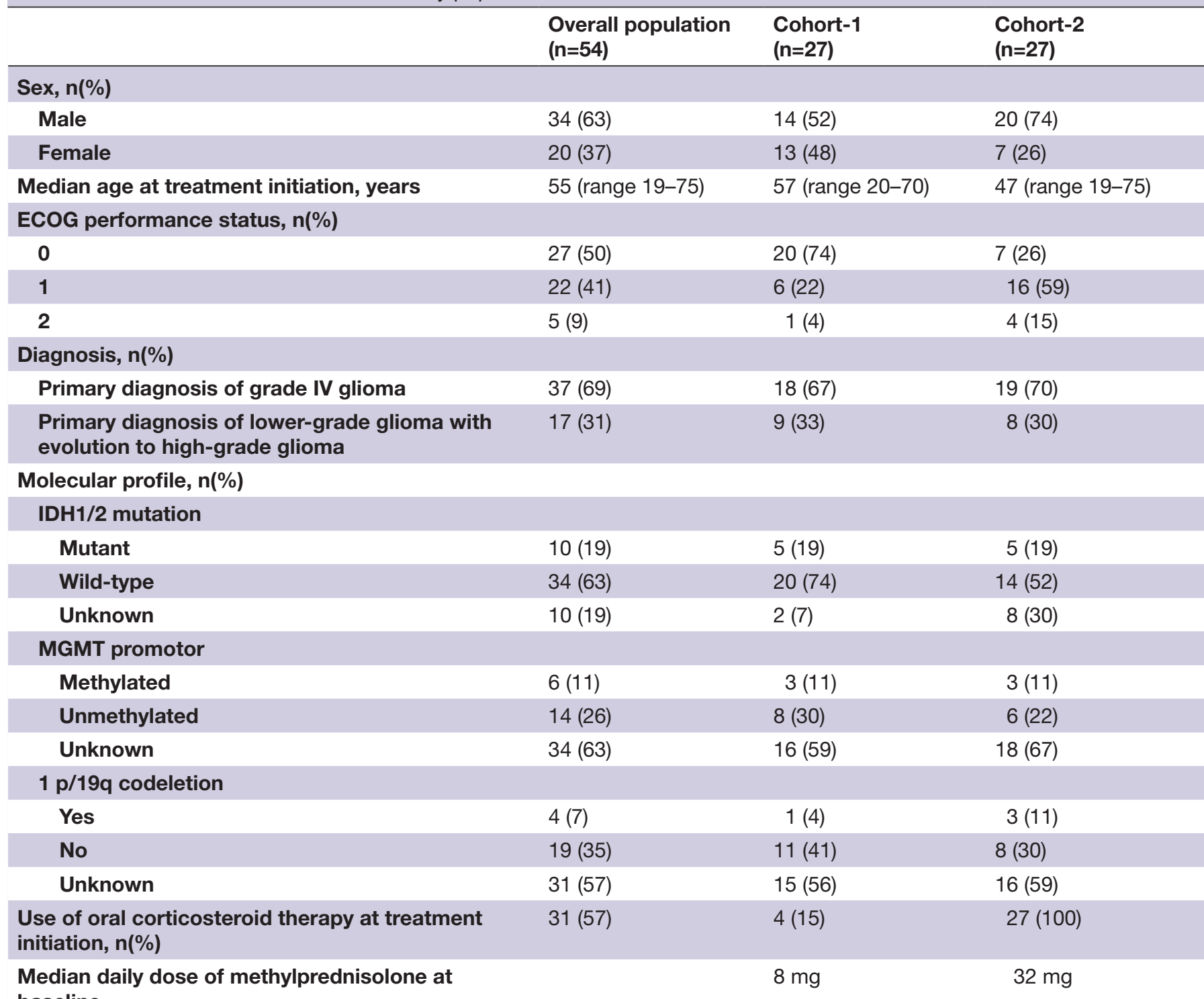

baseline

Prior therapy for primary diagnosis of glioma, $n(\%)$

Primary surgery for diagnosis

$\begin{array}{llll}\begin{array}{l}\text { Resection } \\ \text { Biopsy }\end{array} & 47(87) & 24(89) & 23(85) \\ \begin{array}{l}\text { Primary therapeutic strategy } \\ \text { Resection/biopsy only }\end{array} & 7(13) & 3(11) & 4(15) \\ \text { Radiation therapy only } & 7(13) & 0(0) & 1(4) \\ \text { Chemotherapy only } & 1(2) & 0(0) & 1(4) \\ \text { Surgery+chemotherapy only } & 1(2) & 1(4) & 0(0) \\ \text { Surgery+radiation therapy only } & 1(2) & 2(7) & 1(4) \\ \text { Surgery+radiation therapy +chemotherapy } & 1(2) & 0(0) & 1(4) \\ \text { (sequential) } & & 21(78) & 19(70) \\ \text { Surgery+concomitant radiation therapy/ } & 40(74) & 20(74) & 19(70) \\ \text { TMZ+adjuvant TMZ } & & 18(33) & 15(56)\end{array}$

Continued 


\begin{tabular}{|c|c|c|c|}
\hline & $\begin{array}{l}\text { Overall population } \\
(n=54)\end{array}$ & $\begin{array}{l}\text { Cohort-1 } \\
(n=27)\end{array}$ & $\begin{array}{l}\text { Cohort-2 } \\
(n=27)\end{array}$ \\
\hline Median no of surgeries & 1 (range 1-4) & 1 (range 1-2) & 1 (range 1-4) \\
\hline Systemic therapy & $33(61)$ & $16(59)$ & $17(63)$ \\
\hline Median no of lines of systemic therapy & 1 (range $1-4)$ & 1 (range $1-4)$ & 1 (range $1-3$ ) \\
\hline
\end{tabular}

$1 p / 19 q$ codeletion: deletion of short arm of chromosome one and long arm of chromosome 19. ECOG, Eastern Cooperative Oncology Group; GB, glioblastoma; IDH, isocitrate dehydrogenase; MGMT, O-6-methylguanine-DNA methyltransferase; TMZ, temozolomide.

ECOG PS of 0-1 (91\%), 5 patients (one in Cohort-1 and 4 in Cohort-2) had an ECOG PS of 2. Thirty-seven patients $(69 \%)$ had a primary diagnosis of GB, while 17 patients $(31 \%)$ had a primary diagnosis of a lower-grade glioma that had transformed to a GB. In Cohort-1, 4 patients $(15 \%)$ were treated at BL with a daily dose of methylprednisolone $8 \mathrm{mg}$. In Cohort-2, all patients were treated with oral corticosteroids at BL at a median daily dose of $32 \mathrm{mg}$ of methylprednisolone (equivalent of dexamethasone 6 $\mathrm{mg})$.

Previously, most patients in Cohort-1 and Cohort-2 had undergone a resection of their GB at primary diagnosis and had subsequently been treated with adjuvant concomitant radiation therapy and temozolomide, followed by adjuvant temozolomide $(78 \%$ of patients in Cohort-1 and in $70 \%$ of patients in Cohort-2). Seventy-two per cent of patients had also received prior therapies for recurrent disease. Respectively 33\% and $56 \%$ of patients in Cohort-1 and Cohort-2 had undergone at least one resection (median 1 (range 1-4)) for rGB. Radiation therapy for rGB had been administered to six patients in Cohort-1 and five patients in Cohort-2. Prior systemic therapies for recurrence were administered to $59 \%$ of patients in Cohort-1 and to $63 \%$ of patients in Cohort-2.

\section{Treatment disposition}

At the date of analysis (May 19 2020), all patients had permanently discontinued study treatment. The median duration of follow-up after treatment initiation was 24.9 weeks (range 5.3-141.9).

All patients in Cohort-1 and -2 initiated axitinib treatment, and all patients in Cohort-1 initiated avelumab concomitantly. The median duration of axitinib treatment was 19.1 weeks (range 4.0-88.3) in Cohort-1 and 12.0 weeks in Cohort-2 (range 0.7-59.0). The daily dose of axitinib was escalated to 7 or $10 \mathrm{mg}$ two times a day in 13 patients (10 in Cohort- 1 and 3 in Cohort-2). Axitinib dosing needed to be decreased to less than $5 \mathrm{mg}$ two times a day in 11 patients (six in Cohort-1 and five in Cohort-2). A temporary interruption of axitinib dosing was needed in $12(44 \%)$ patients in Cohort-1 (toxicity: nine patients; non-rGB-/non-treatment-related events: 3 patients). There were no permanent discontinuations of axitinib due to toxicity in Cohort-1. Sixteen patients in Cohort-2 (59\%) temporarily interrupted axitinib dosing (toxicity: 13 patients; non-rGB-/non-treatment-related events: 3 patients). Five patients (18\%) in Cohort-2 permanently discontinued axitinib for reasons other than progressive disease (three patients because of pulmonary embolism, one patient because of microscopic colitis and one patient because of infectious meningitis).

All patients in Cohort-1 initiated avelumab at the same time as axitinib. Median duration of avelumab treatment in Cohort-1 was 14 weeks (range 2.0-72.0). Seventeen (63\%) patients in Cohort-2 initiated avelumab after reducing their daily dose of corticosteroids to an equivalent of $8 \mathrm{mg}$ of methylprednisolone or less: 13 patients reduced the dose after 6 weeks of axitinib monotherapy and 4 patients were allowed to do so at a later point in time because of incomplete steroid tapering after 6 weeks $(n=3)$ or pneumonia $(n=1)$. Ten patients $(37 \%)$ in Cohort-2 never initiated avelumab treatment because of early progressive disease (seven patients), axitinib monotherapy-related toxicity leading to early permanent discontinuation (two patients with pulmonary embolism), or a severe infection (infectious meningitis) leading to permanent axitinib discontinuation in one additional patient. Median duration of avelumab treatment in Cohort-2 was 6.0 weeks (range 0.0-34.0).

Avelumab dosing was temporarily interrupted in seven $(26 \%)$ patients in Cohort-1 (toxicity: three patients; nonrGB-/non-treatment-related events: four patients) and permanently discontinued in 1 (grade 3 immune-related hepatitis and pneumonitis).

Out of the 17 patients in Cohort-2 who initiated treatment with avelumab, temporary interruptions were necessary in 12 patients $(71 \%)$ (because of corticosteroid need leading to incompatibility with avelumab administration (six patients), non-rGB-/non-treatment-related events (five patients) and a microscopic colitis in one additional patient). Avelumab was permanently discontinued in five of these patients (one patient with pulmonary embolism, one patient with immune-related diarrhea and microscopic colitis, one patient with paraesthesia and two patients with a permanent need for supraphysiological corticosteroid doses).

\section{Efficacy}

In the overall population 15 out of 54 patients $(27.8 \%)$ achieved a confirmed complete or partial response per 
Table 2 Objective response and disease control rate (per iRANO) in the study population

\begin{tabular}{llll}
\hline & $\begin{array}{l}\text { Overall population } \\
(\mathbf{n}=\mathbf{5 4})\end{array}$ & $\begin{array}{l}\text { Cohort-1 } \\
(\mathbf{n = 2 7 )}\end{array}$ & $\begin{array}{l}\text { Cohort-2 } \\
(\mathbf{n}=\mathbf{2 7})\end{array}$ \\
\hline $\begin{array}{l}\text { Confirmed objective response, } \mathbf{n}(\%) \\
\text { Complete response }\end{array}$ & $15(27.8 \%)$ & $9(33.3 \%)$ & $6(22.2 \%)$ \\
\hline Partial response & $2(3.7 \%)$ & $1(3.7 \%)$ & $1(3.7 \%)$ \\
\hline Stable disease, $\mathbf{n}(\%)$ & $13(24.1 \%)$ & $8(29.6 \%)$ & $5(18.5 \%)$ \\
\hline Progressive disease, $\mathbf{n}(\%)$ & $15(27.8 \%)$ & $8(29.6 \%)$ & $7(25.9 \%)$ \\
\hline Disease control rate, $\mathbf{n}(\%)$ & $24(44.4 \%)$ & $10(37.0 \%)$ & $14(51.9 \%)$ \\
\hline Median duration of response, weeks & $30(55.6 \%)$ & $17(63.0 \%)$ & $13(48.1 \%)$ \\
\hline Median time to response, weeks & 18.0 & 17.9 & 19.0 \\
$($ range 6.0-83.1) & $($ range 6.0-83.1) & $($ range 8.0-38.0) \\
\hline
\end{tabular}

iRANO, immunotherapy response assessment for neuro-oncology.

iRANO criteria (table 2). In Cohort-1 the confirmed ORR was $33.3 \%$ (one confirmed complete $(3.7 \%)$, and eight confirmed partial responses $(29.6 \%)$ were observed) (figure 2). The median DOR in cohort 1 was 17.9 weeks (range 6.0-83.1). In Cohort-2 the confirmed ORR was $22.2 \%$ (one confirmed complete $(3.7 \%)$, and five confirmed partial response $(18.5 \%)$ ). The median DOR was 19.0 weeks (range 8.0-38.0). In patients who achieved a confirmed OR, the median time to response was 6.0 weeks (range 5.1-18.0) in Cohort-1 and 5.9 weeks (range 4.7-8.0) in Cohort-2. The disease control rate was $63.0 \%$ and $48.1 \%$ in Cohort-1 and Cohort-2, respectively.

At the time of analysis (May 19 2020), all patients have progressed and 50 have died. The median follow-up of the four surviving patients is 114.9 weeks (range 100.9141.9 ). The 6 -m-PFS\% is $22.2 \%$ (95\% CI $6.5 \%$ to $37.9 \%$ ), and $18.5 \%(95 \%$ CI $3.8 \%$ to $33.2 \%)$ in Cohort- 1 and Cohort-2, respectively (figures 3 and 4). The median PFS is 12.0 (95\% CI 8.2 to 15.8 ), and 10.7 weeks (95\% CI 5.3 to 16.1 ), respectively. The median OS is 26.6 weeks (95\% CI 20.8 to 32.4 ) and the OS rate at 12 months (52 weeks) is $22.2 \%$ (95\% CI 6.5\% to $37.9 \%$ ) in Cohort-1; in Cohort-2, the median OS is 18.0 weeks (95\% CI 12.5 to 23.5 ) and the OS rate at 12 months $11.1 \%$ (95\% CI $0 \%$ to $22.9 \%$ ).

\section{Safety}

Treatment-related adverse events (TRAEs) were observed in all patients (table 3). Most TRAEs were low grade and reversible and no grade 5 TRAEs events occurred. The most common TRAEs were dysphonia (67\%, all grade), lymphopenia $(50 \%$, all grade), diarrhea and arterial hypertension (both $48 \%$, all grade), fatigue $(46 \%$, all grade) and mucositis (24\%, all grade). Grade 1 or 2 avelumab infusion reactions, chills and/or fever during or immediately after the infusion of avelumab, were seen in $11 \%$, and $9 \%$ of patients, respectively. Adverse events of special interest were pulmonary embolism $(6 \%$, all grade $4)$, immune-related hepatitis (4\%; 1 grade 2,1 grade 3$)$, immune-related pneumonitis (2\%; grade 3 ), psoriasiform rash (2\%; grade 2$)$ and microscopic colitis (2\%, grade 2$)$.

\section{HRQOL and neurocognitive function}

All patients were assessed for HRQoL and neurocognitive function at BL. At week 9 (follow-up 1), and week

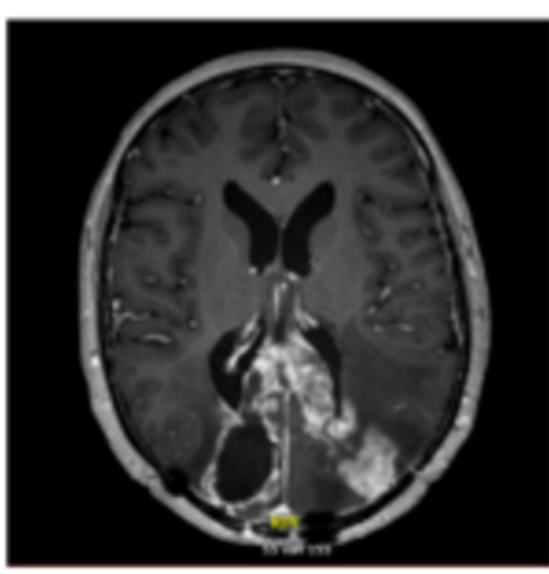

Baseline

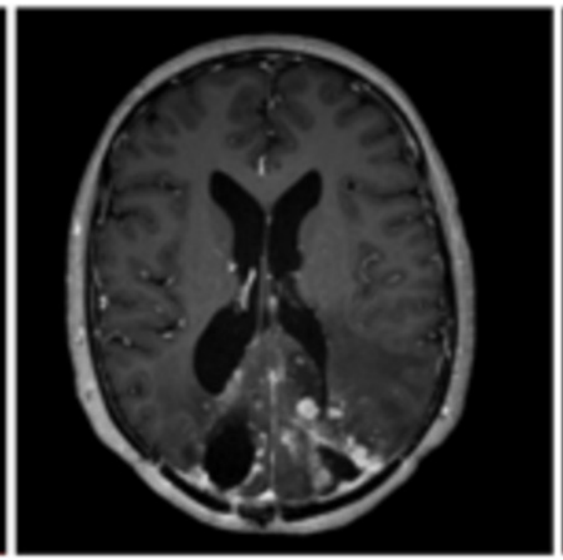

Week 6

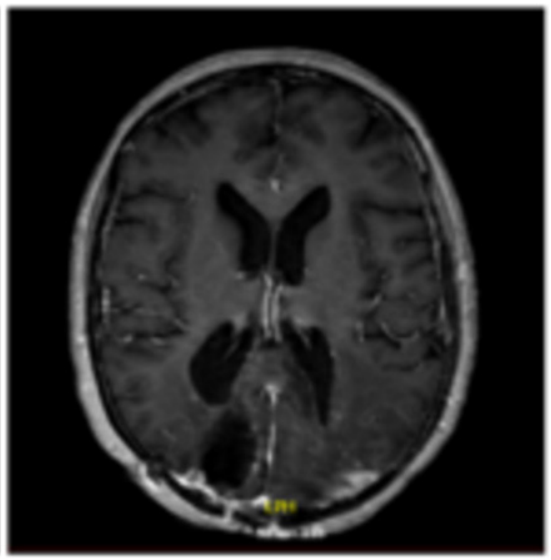

Week 12

Figure 2 Gadolinium-enhanced T1 MR images of a study patient treated with axitinib and avelumab who developed a partial response (Cohort-1). 
A

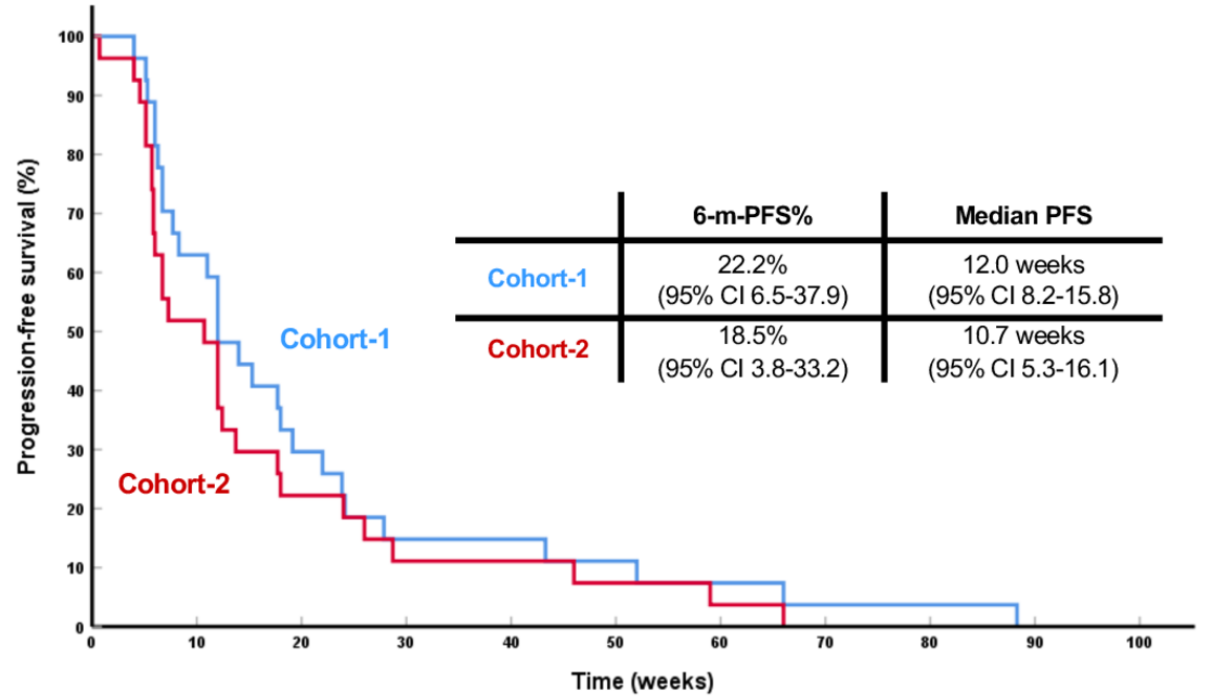

\begin{tabular}{|l|c|c|c|c|c|c|c|c|c|c|}
\hline $\begin{array}{c}\text { Number at } \\
\text { risk } \\
\text { (number } \\
\text { censored) }\end{array}$ & $0 \mathrm{w}$ & $10 \mathrm{w}$ & $\mathbf{2 0} \mathrm{w}$ & $\mathbf{3 0 w}$ & $\mathbf{4 0 w}$ & $\mathbf{5 0 w}$ & $\mathbf{6 0} \mathrm{w}$ & $\mathbf{7 0 w}$ & $\mathbf{8 0 \mathrm { w }}$ & $\mathbf{9 0 \mathrm { w }}$ \\
\hline Cohort-1 & $27(0)$ & $17(0)$ & $8(0)$ & $4(0)$ & $4(0)$ & $3(0)$ & $2(0)$ & $1(0)$ & $1(0)$ & $0(0)$ \\
\hline Cohort-2 & $27(0)$ & $14(0)$ & $6(0)$ & $3(0)$ & $3(0)$ & $2(0)$ & $1(0)$ & $0(0)$ & $0(0)$ & $0(0)$ \\
\hline
\end{tabular}

B

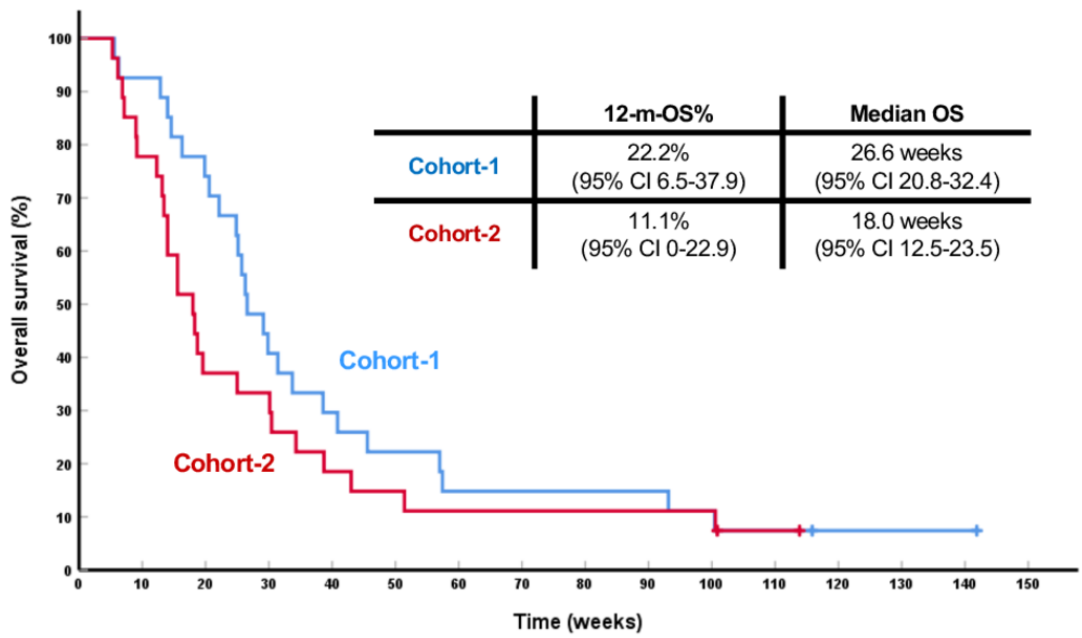

\begin{tabular}{|c|c|c|c|c|c|c|c|c|c|c|c|c|c|c|c|c|}
\hline $\begin{array}{l}\text { Number at } \\
\text { risk } \\
\text { (number } \\
\text { censored) }\end{array}$ & $\stackrel{0}{<}$ & $\begin{array}{l}\text { ठे } \\
\Sigma\end{array}$ & $\begin{array}{l}\text { N } \\
\Sigma\end{array}$ & $\begin{array}{l}\omega \\
\Sigma\end{array}$ & $\begin{array}{l}\text { ठ } \\
\Sigma\end{array}$ & $\begin{array}{l}\text { CI } \\
\text { < }\end{array}$ & $\begin{array}{l}8 \\
<\end{array}$ & $\begin{array}{l}\text { ठ } \\
\Sigma\end{array}$ & $\begin{array}{l}\text { ø } \\
\text { < }\end{array}$ & $\begin{array}{l}\varnothing \\
\Sigma\end{array}$ & $\begin{array}{l}\overrightarrow{8} \\
\Sigma\end{array}$ & $\begin{array}{l}\overrightarrow{\overrightarrow{0}} \\
\Sigma\end{array}$ & $\begin{array}{l}\vec{N} \\
\Sigma\end{array}$ & $\begin{array}{l}\vec{\omega} \\
\Sigma\end{array}$ & $\begin{array}{l}\overrightarrow{\hat{\theta}} \\
\Sigma\end{array}$ & $\begin{array}{l}\text { ज्ञ } \\
\text { ₹ }\end{array}$ \\
\hline Cohort-1 & $\begin{array}{l}27 \\
(0)\end{array}$ & $\begin{array}{l}25 \\
(0)\end{array}$ & $\begin{array}{l}20 \\
(0)\end{array}$ & $\begin{array}{l}11 \\
(0)\end{array}$ & $\begin{array}{c}8 \\
(0)\end{array}$ & $\begin{array}{c}6 \\
(0)\end{array}$ & $\begin{array}{c}4 \\
(0)\end{array}$ & $\begin{array}{c}4 \\
(0)\end{array}$ & $\begin{array}{c}4 \\
(0)\end{array}$ & $\begin{array}{c}4 \\
(0)\end{array}$ & $\begin{array}{c}3 \\
(0)\end{array}$ & $\begin{array}{c}2 \\
(0)\end{array}$ & $\begin{array}{c}2 \\
(1)\end{array}$ & $\begin{array}{c}2 \\
\text { (1) }\end{array}$ & $\begin{array}{c}2 \\
(1)\end{array}$ & $\begin{array}{c}2 \\
(2)\end{array}$ \\
\hline Cohort-2 & $\begin{array}{l}27 \\
(0)\end{array}$ & $\begin{array}{l}21 \\
(0)\end{array}$ & $\begin{array}{l}10 \\
(0)\end{array}$ & $\begin{array}{c}9 \\
(0)\end{array}$ & $\begin{array}{c}5 \\
(0)\end{array}$ & $\begin{array}{l}23 \\
(0)\end{array}$ & $\begin{array}{c}3 \\
(0)\end{array}$ & $\begin{array}{c}3 \\
(0)\end{array}$ & $\begin{array}{c}3 \\
(0)\end{array}$ & $\begin{array}{c}3 \\
(0)\end{array}$ & $\begin{array}{c}3 \\
(0)\end{array}$ & $\begin{array}{c}2 \\
(1)\end{array}$ & $\begin{array}{c}2 \\
(2)\end{array}$ & & & \\
\hline
\end{tabular}

Figure 3 PFS and OS curves. (A) PFS in Cohort-1 (blue) and Cohort-2 (red) from the start of study drugs. (B) OS in cohort 1 (blue) and cohort 2 (red) from the start of study drugs. 6-m-PFS\%: 6-month-PFS rate; 12-m-OS\%: OS rate at 12 months (52 weeks); $95 \% \mathrm{Cl}:$ 95\% confidence interfal.

21 (follow-up 2), respectively, 35 and 18 patients were eligible for assessment. At BL, Cohort-1 had a better global HRQoL, physical, social and role functioning but had higher levels of fatigue and motor dysfunction symptoms compared with Cohort-2. At follow-up 1, Cohort-2 had higher symptom levels of nausea and vomiting, 
A

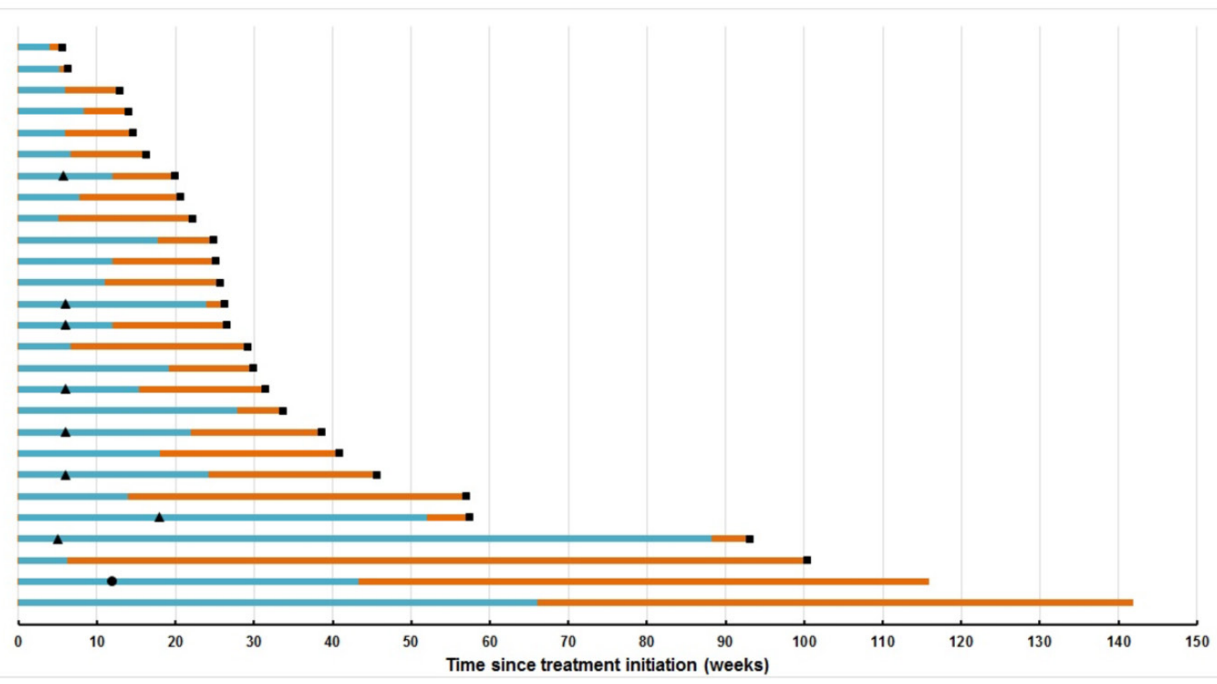

B

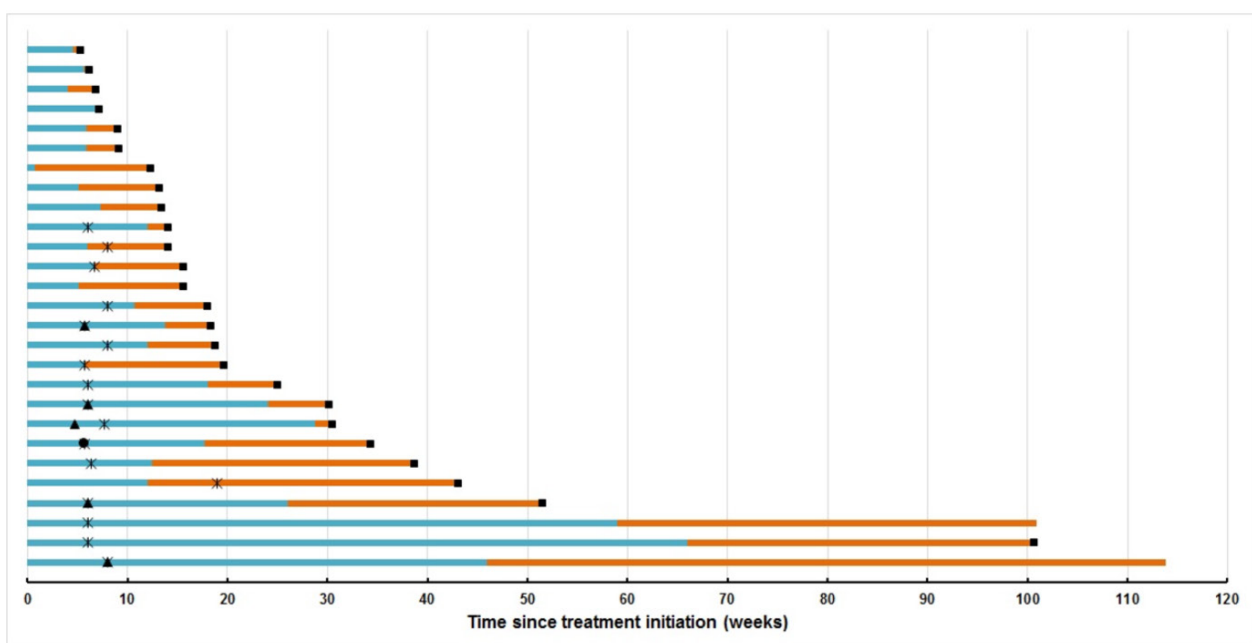

Figure 4 Swimmer plots. (A) Swimmer plot depicting progression-free (blue) and overall survival (orange) of patients in Cohort-1 treated with avelumab and axitinib; (B) swimmer plot depicting progression-free survival (blue) and overall survival (orange) of patients in Cohort-2. Black circle denotes timepoint of complete response, black triangle denotes timepoint of partial response, black square denotes timepoint of death. The black cross denotes the start of avelumab in Cohort-2 patients.

financial difficulties and drowsiness. No significant differences in neurocognitive functioning were found at $\mathrm{BL}$ between Cohort-1 and Cohort-2. Descriptive statistics at BL of HRQoL and cognitive function are summarized in online supplementary table S3. In general, BL differences between the cohorts remained stable at follow-up. Processing speed, attention and working memory significantly deteriorated at follow-up 1 and follow-up 2.

\section{DISCUSSION}

This prospective phase 2 trial failed to meet its primary objective in demonstrating a $6-\mathrm{m}-\mathrm{PFS} \%$ of more than $50 \%$ in patients with rGB without need for steroid treatment on treatment with the oral small molecule VEGFRinhibitor axitinib in combination with the PD-L1 blocking monoclonal antibody avelumab. Neither did this study provide a signal considered worthy of further investigation of this combinatorial treatment in patients with rGB, independently of whether patients needed corticosteroid treatment at the initiation of study treatment.

All endpoints assessing the activity of the investigational combination therapy are considered not to be meaningfully different from the results previously obtained by our group in two recently reported phase 2 clinical trials with axitinib monotherapy or the combination of axitinib and lomustine. ${ }^{89}$ The observed activity is therefore likely to result from the previously reported activity of axitinib monotherapy, resulting in a transient disease control rate that is comparable to results obtained with bevacizumab or cediranib-based regimens for rGB. ${ }^{1617}$ 
Table 3 Treatment-related adverse events

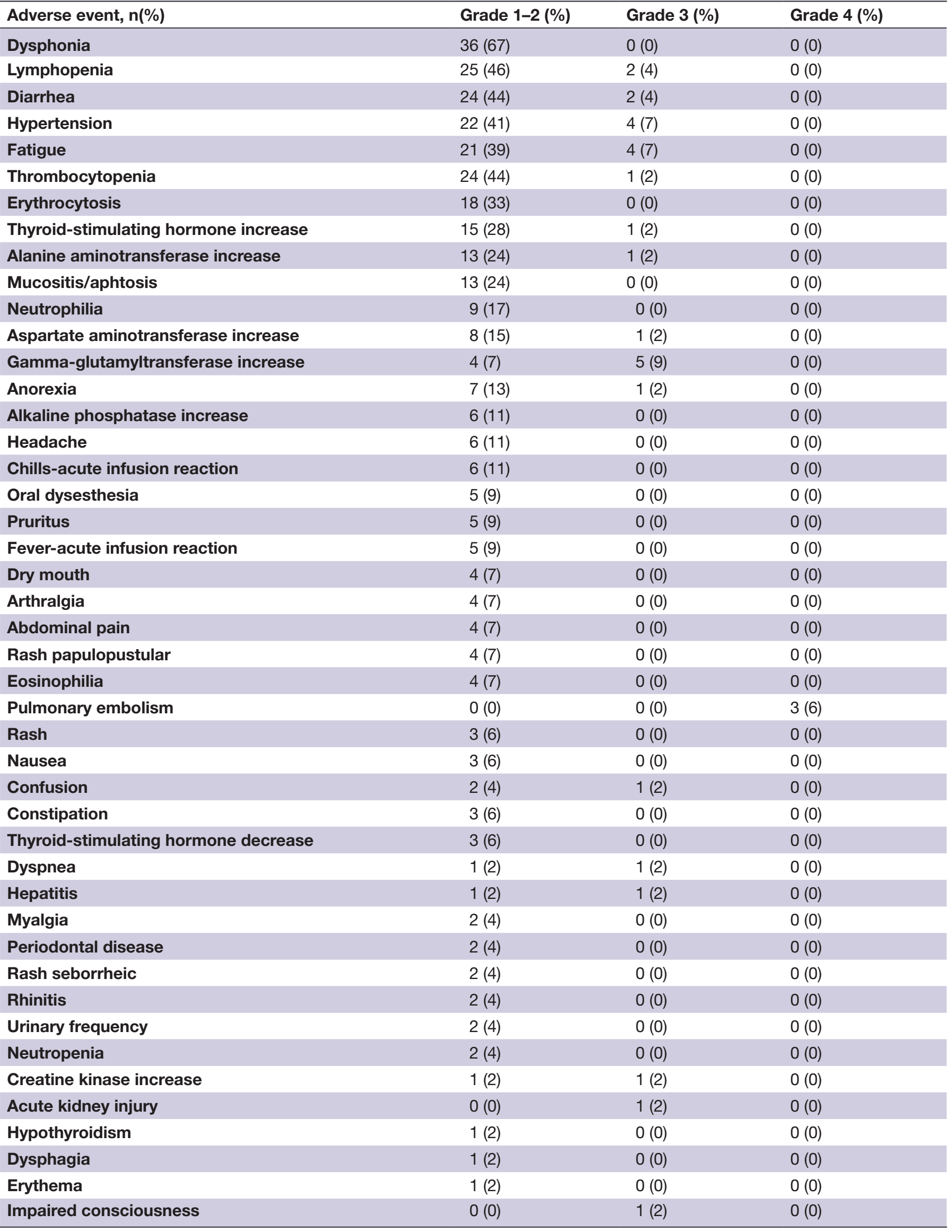


Table 3 Continued

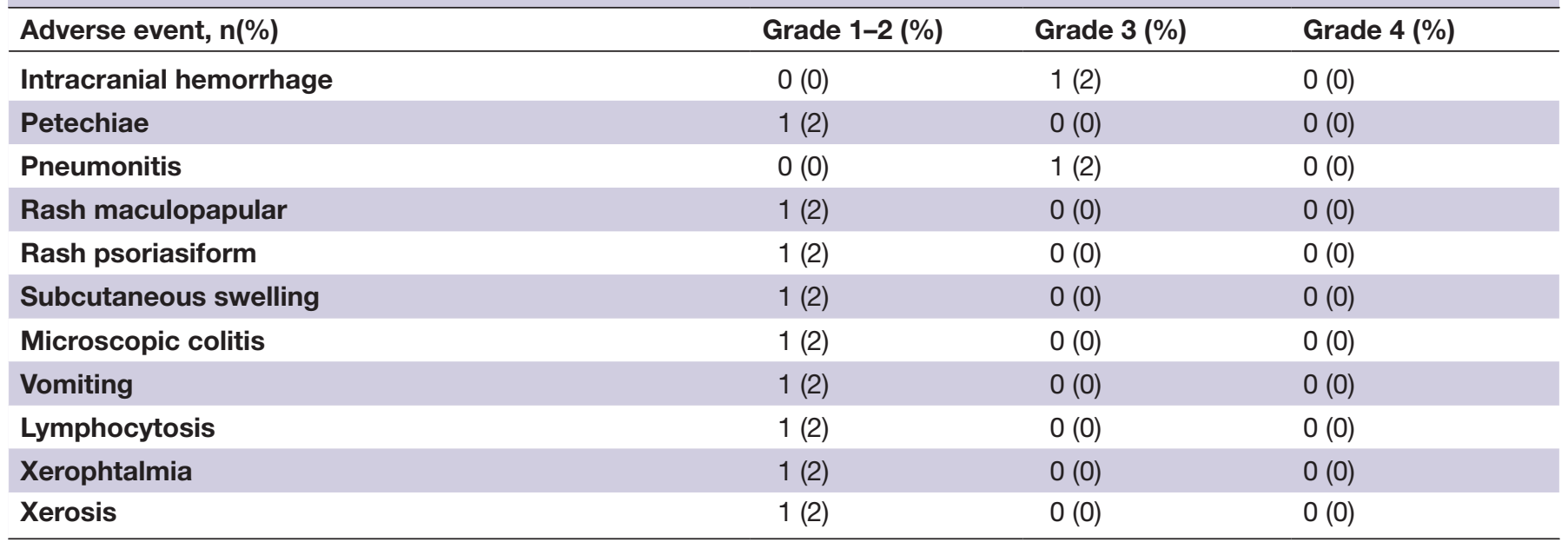

Our results indicating the absence of a convincing level of activity of the PD-L1 blocking monoclonal antibody avelumab are in line with the previously reported sobering results obtained with the PD-1 blocking antibodies nivolumab and pembrolizumab in rGB (either as monotherapy or in combination with bevacizumab). ${ }^{11} 18$ Despite reported high PD-L1 expression in primary and $\mathrm{rGB}$, the GB tumor microenvironment is characterized by a low frequency of exhausted tumor-infiltrating lymphocytes with a high level of co-expression of other inhibitory immune checkpoints (CTLA4, lymphocyte-activation gene 3 and T-cell immunoglobulin mucin-3) which PD1/ PD-L1 blocking monoclonal antibodies may not be able to overcome. ${ }^{1920}$ In addition, expression of the B7-H3 (B7 homolog 3) immune checkpoint has been associated with glioma-specific immunosuppression. ${ }^{21}$ The durability of response is often considered a hallmark of cancer immune checkpoint inhibition and previously reported for a small proportion of patients with rGB treated with nivolumab in the Checkmate-143 phase 3 trial. This was, however, seemingly not improved as compared with historical controls in our trial as all patients with a confirmed objective response eventually progressed. Absence of highly durable tumor responses withheld us from investigating potential predictors of benefit from treatment with axitinib and avelumab such as tumor mutation burden, PD-L1 immunohistochemical score, or T-cell inflamed gene expression signatures.

Our results indicate that in contrast to advanced renal cell carcinoma where the combination of avelumab and axitinib has shown synergistic activity and superior results to the multitargeted kinase inhibitor sunitinib, this combination may not be sufficient to overcome immune suppression within the GB tumor microenvironment resulting in a superior clinical activity in patients with rGB. $^{14}$

The results of this trial confirm our earlier phase 2 study reports that axitinib, by efficiently reducing tumorassociated edema, allowed to taper corticosteroid dose in 17 out of 27 patients in Cohort-2 without worsening of neurological symptoms. ${ }^{8} 9$ Axitinib could, therefore, still be considered a potentially useful agent in future immunotherapy regimens for rGB, allowing patients to safely reduce or stop immunosuppressive corticosteroid treatment before embarking on immunotherapy. The short half-life of axitinib could be a safety advantage when there is a potential need for interrupting treatment in case of side effects.

Treatment with avelumab and axitinib was generally well tolerated and no unexpected TRAEs were seen. AEs were mostly of low grade and manageable and reversible with supportive therapy, temporary corticosteroid treatment (for immune-related AEs), and temporary treatment interruption or dose reduction. The incidence of avelumab-related immune-related AEs was lower than seen in other tumor types. This may relate to the systemically immunosuppressed state of patients with rGB following long periods of corticosteroid treatment. The few cases diagnosed with immune-related AEs do indicate the biological activity of avelumab in this patient population. Potentially life-threatening AEs consisted of three patients with pulmonary embolism, and one patient with intracranial hemorrhage. While these cases highlight the need for increased cautiousness in patients considered at risk for such events, their incidence is not clearly higher that what can be expected in patients with $\mathrm{rGB}$ treated with other modalities.

With respect to the EORTC QLQ-C30-BN20 instrument, Dirven $e t$ al and Taphoorn et al reported that global QoL, physical functioning, social functioning, motor dysfunction and communication deficit were the most relevant subscales for assessing HRQoL in GB patients. ${ }^{22} 23$ Consistently, we found a better global HRQoL, physical and social functioning in the patients who were not in need of corticotherapy at BL (Cohort-1), and higher prevalence of motor dysfunction in those who were (Cohort-2). This difference remained stable during follow-up at week 9 and 21, notwithstanding the steroid sparing effect of the study treatment. 
Based on computerized cognitive assessments performed in between tumor response assessments at week 9 and 21, a significant deterioration in 4 out of 5 domains of investigated neurocognitive function was observed. Deterioration during study treatment was also observed for the cognitive functioning scale of the EORTC QLQ-C30, this finding however was not statistically significant. This observation is in line with the findings of Gilbert $e t a l$ who reported a deterioration of objective and subjective neurocognitive functioning in newly diagnosed GB patients treated with VEGF-blocking monoclonal antibody bevacizumab in combination with radiation therapy. ${ }^{24}$ Our observations are therefore indicative for the fact that neurocognitive function may be at risk of deterioration while disease progression is absent or unrecognized on conventional MRI assessment of tumor response in GB patients treated with agents inhibiting the VEGF-/ VEGFR-axis.

A limitation that should be considered when extrapolating our study results are the BL characteristics of our study population which consisted mostly of patients with primary GB of which many were extensively pretreated. Most patients $(72 \%)$ had received one or more treatment modalities for recurrent disease prior to treatment initiation within this study. This could have impacted negatively on their chances to benefit from PD-L1 immune checkpoint therapy as the activity of PD-1/PD-L1 targeted immunotherapy diminishes with increasing lines of prior therapy in other cancer types such as melanoma. ${ }^{25}{ }^{26}$ Tolerability is less likely to have impacted on treatment outcome, especially in the patients who initiated axitinib plus avelumab concomitantly (Cohort1). Temporary or permanent interruption of the study drugs was infrequent in patients treated in Cohort-1. It was higher in patients treated in Cohort-2 and 10 out of 27 patients were never treated with avelumab, indicating the more difficult setting of addressing the needs of patients who have become dependent on corticosteroids to control disease related symptoms.

In conclusion, the combination of avelumab and axitinib did not meet its threshold for activity in an unselected population of adult patients with rGB, regardless of their BL use of corticosteroids. We were not able to identify a subpopulation of patients where this combination could be of benefit. The efficacy of the combination is similar to our previous experience with axitinib monotherapy and the addition of avelumab does not provide a signal of synergistic efficacy. Axitinib confirms its role as a potent corticoid-sparing and corticoid-tapering agent to control tumor-related edema. No unexpected AEs were seen with axitinib monotherapy or with the combination of axitinib and avelumab. Further research is clearly warranted for this indication as patients with rGB are in need of better treatment options. A better understanding of the immunobiology of $\mathrm{GB}$ will be needed in order to rationally conceive combinatorial regimens with a higher chance of success.

\section{Author affiliations}

${ }^{1}$ Medical Oncology, Universitair Ziekenhuis Brussel, Brussels, Belgium

${ }^{2}$ Psychology, Vrije Universiteit Brussel, Brussels, Brussels, Belgium
${ }^{3}$ Neurology, Universitair Ziekenhuis Brussel, Brussels, Belgium ${ }^{4}$ Neurosurgery, Universitair Ziekenhuis Brussel, Brussels, Belgium ${ }^{5}$ Radiology, Universitair Ziekenhuis Brussel, Brussels, Belgium ${ }^{6}$ Pathology, Universitair Ziekenhuis Brussel, Brussels, Belgium ${ }^{7}$ Nuclear Medicine, Universitair Ziekenhuis Brussel, Brussels, Belgium ${ }^{8}$ Psychiatry, Centre Hospitalier Universitaire Brugmann, Brussels, Belgium

Acknowledgements The authors would like to thank the patients and their families who enrolled on this clinical trial, along with the clinical research staff, in particular Katrien Van Den Bossche and Katrien Van Peteghem.

Contributors Study design: BN, JD, SDF and AR. Implementation: all authors. Analysis, interpretation of data: GA, LBS, JDC, LF, AR, PT, JD and BN. Writing of the manuscript: GA, LBS, JDC, AR, PT and BN. Revision of the manuscript: all authors.

Funding This study was designed and funded by the Universitair Ziekenhuis Brussel. Pfizer provided study drugs (avelumab and axitinib) free of charge. The corresponding author had full access to all the data in the study and had final responsibility for the decision to submit for publication. The manuscript was reviewed and approved by Pfizer prior to submission to this journal.

Disclaimer Pfizer had no role in the study design, data collection, data analysis, data interpretation or writing of the report and did not have access to the raw data.

Competing interests GA reports non-financial support from MSD, non-financial support from Astellas, non-financial support from Novartis outside the submitted work. JKS reports non-financial support from Amgen, non-financial support from MSD outside the submitted work. AR reports personal fees from Bristol-Myers Squibb, personal fees from MSD outside the submitted work.Dr. Neyns reports nonfinancial support from Pfizer, non-financial support from Merck Serono during the conduct of the study; personal fees from Roche, personal fees from Bristol-Myers Squibb, personal fees from MSD, personal fees from Novartis, personal fees from AstraZeneca, personal fees from eTheRNA, personal fees from CryoStorage, grants from Pfizer, grants from Merck Serono, grants from Novartis, grants from Roche, outside the submitted work. All remaining authors have declared no conflicts of interest.

Patient consent for publication Not required.

Ethics approval The study was done in accordance with both the Declaration of Helsinki and the International Conference on Harmonization Good Clinical Practice guidelines and was approved by the institutional medical ethics committee of the Universitair Ziekenhuis Brussel (0.G. 016) and by the Belgian Federal Agency for Medicines and Health Products.

Provenance and peer review Not commissioned; externally peer reviewed.

Data availability statement All data relevant to the study are included in the article or uploaded as online supplemental information. The datasets used and/or analyzed during the current study are available from the corresponding author on reasonable request.

Supplemental material This content has been supplied by the author(s). It has not been vetted by BMJ Publishing Group Limited (BMJ) and may not have been peer-reviewed. Any opinions or recommendations discussed are solely those of the author(s) and are not endorsed by BMJ. BMJ disclaims all liability and responsibility arising from any reliance placed on the content. Where the content includes any translated material, BMJ does not warrant the accuracy and reliability of the translations (including but not limited to local regulations, clinical guidelines, terminology, drug names and drug dosages), and is not responsible for any error and/or omissions arising from translation and adaptation or otherwise.

Open access This is an open access article distributed in accordance with the Creative Commons Attribution Non Commercial (CC BY-NC 4.0) license, which permits others to distribute, remix, adapt, build upon this work non-commercially, and license their derivative works on different terms, provided the original work is properly cited, appropriate credit is given, any changes made indicated, and the use is non-commercial. See http://creativecommons.org/licenses/by-nc/4.0/.

\section{ORCID iD}

Gil Awada http://orcid.org/0000-0001-9535-0430

\section{REFERENCES}

1 Stupp R, Hegi ME, Mason WP, et al. Effects of radiotherapy with concomitant and adjuvant temozolomide versus radiotherapy alone 
on survival in glioblastoma in a randomised phase III study: 5-year analysis of the EORTC-NCIC trial. Lancet Oncol 2009;10:459-66.

2 Wong ET, Hess KR, Gleason MJ, et al. Outcomes and prognostic factors in recurrent glioma patients enrolled onto phase II clinical trials. J Clin Oncol 1999;17:2572-8.

3 Lamborn KR, Yung WKA, Chang SM, et al. Progression-Free survival: an important end point in evaluating therapy for recurrent high-grade gliomas. Neuro Oncol 2008;10:162-70.

4 Szabo E, Schneider H, Seystahl K, et al. Autocrine VEGFR1 and VEGFR2 signaling promotes survival in human glioblastoma models in vitro and in vivo. Neuro Oncol 2016;18:1242-52.

5 Friedman HS, Prados MD, Wen PY, et al. Bevacizumab alone and in combination with irinotecan in recurrent glioblastoma. J Clin Oncol 2009;27:4733-40.

6 Kelly RJ, Rixe O. Axitinib (AG-013736). Recent Results Cancer Res 2010;184:33-44.

7 Lu L, Saha D, Martuza RL, et al. Single agent efficacy of the VEGFR kinase inhibitor axitinib in preclinical models of glioblastoma. $J$ Neurooncol 2015;121:91-100.

8 Duerinck J, Du Four S, Vandervorst F, et al. Randomized phase II study of axitinib versus physicians best alternative choice of therapy in patients with recurrent glioblastoma. J Neurooncol 2016:128:147-55.

9 Duerinck J, Du Four S, Bouttens F, et al. Randomized phase II trial comparing axitinib with the combination of axitinib and lomustine in patients with recurrent glioblastoma. J Neurooncol 2018;136:115-25.

10 Ribas A, Wolchok JD. Cancer immunotherapy using checkpoint blockade. Science 2018;359:1350-5.

11 Reardon DA, Omuro A, Brandes AA, et al. OS10.3 randomized phase 3 study evaluating the efficacy and safety of nivolumab vs bevacizumab in patients with recurrent glioblastoma: CheckMate 143. Neuro Oncol 2017;19:iii21.

12 Du Four S, Maenhout SK, De Pierre K, et al. Axitinib increases the infiltration of immune cells and reduces the suppressive capacity of monocytic MDSCs in an intracranial mouse melanoma model. Oncoimmunology 2015;4:e998107.

13 Du Four S, Maenhout SK, Benteyn D, et al. Disease progression in recurrent glioblastoma patients treated with the VEGFR inhibitor axitinib is associated with increased regulatory $T$ cell numbers and $T$ cell exhaustion. Cancer Immunol Immunother 2016;65:727-40.

14 Motzer RJ, Penkov K, Haanen J, et al. Avelumab plus axitinib versus sunitinib for advanced renal-cell carcinoma. $N$ Engl J Med 2019;380:1103-15.
15 Arbour KC, Mezquita L, Long N, et al. Impact of baseline steroids on efficacy of programmed cell death-1 and programmed Death-Ligand 1 blockade in patients with non-small-cell lung cancer. $J$ Clin Oncol 2018;36:2872-8.

16 Batchelor TT, Mulholland P, Neyns B, et al. Phase III randomized trial comparing the efficacy of cediranib as monotherapy, and in combination with lomustine, versus lomustine alone in patients with recurrent glioblastoma. J Clin Oncol 2013;31:3212-8.

17 Wick W, Gorlia T, Bendszus M, et al. Lomustine and bevacizumab in progressive glioblastoma. N Engl J Med 2017;377:1954-63.

18 Reardon DA, Nayak L, Peters KB, et al. Phase II study of pembrolizumab or pembrolizumab plus bevacizumab for recurrent glioblastoma (rGBM) patients. JCO 2018;36:2006.

19 Berghoff AS, Kiesel B, Widhalm G, et al. Programmed death ligand 1 expression and tumor-infiltrating lymphocytes in glioblastoma. Neuro Oncol 2015;17:1064-75.

20 Wherry EJ, Kurachi M. Molecular and cellular insights into T cell exhaustion. Nat Rev Immunol 2015;15:486-99.

21 Zhang $\mathrm{C}$, Zhang Z, Li F, et al. Large-Scale analysis reveals the specific clinical and immune features of $\mathrm{B} 7-\mathrm{H} 3$ in glioma. Oncoimmunology 2018;7:e1461304.

22 Dirven L, van den Bent MJ, Bottomley A, et al. The impact of bevacizumab on health-related quality of life in patients treated for recurrent glioblastoma: results of the randomised controlled phase 2 BELOB trial. Eur J Cancer 2015;51:1321-30.

23 Taphoorn MJB, Henriksson R, Bottomley A, et al. Health-Related quality of life in a randomized phase III study of bevacizumab, temozolomide, and radiotherapy in newly diagnosed glioblastoma. $J$ Clin Oncol 2015;33:2166-75.

24 Gilbert MR, Dignam JJ, Armstrong TS, et al. A randomized trial of bevacizumab for newly diagnosed glioblastoma. N Engl J Med 2014;370:699-708.

25 Weber JS, D'Angelo SP, Minor D, et al. Nivolumab versus chemotherapy in patients with advanced melanoma who progressed after anti-CTLA-4 treatment (CheckMate 037): a randomised, controlled, open-label, phase 3 trial. Lancet Oncol 2015;16:375-84.

26 Ribas A, Puzanov I, Dummer R, et al. Pembrolizumab versus investigator-choice chemotherapy for ipilimumab-refractory melanoma (KEYNOTE-002): a randomised, controlled, phase 2 trial. Lancet Oncol 2015;16:908-18. 